\title{
Primary angiosarcoma of the heart mimicking a left atrial myxoma
}

\author{
OJM ORMEROD, PM SPRATT, NP LEWIS, J WALLWORK \\ From the Departments of Cardiology and Cardiothoracic Surgery, Papworth Hospital, Cambridge
}

Sarcomas as a group are the second most common type of primary tumour of the heart.' Angiosarcoma is, however, an unusual form of tumour within this group and is rarely diagnosed during life. ${ }^{2}$ It is almost exclusively a tumour of the right atrium and pericardium, patients presenting with symptoms of right heart inflow obstruction and pericardial disease. ${ }^{3}$ We present here a case of primary angiosarcoma of the heart causing obstruction of the mitral valve. Two dimensional echocardiography suggested the presence of a left atrial myxoma. Resection was attempted. The histological diagnosis was made from tissue obtained at surgery.

\section{Case report}

A 48 year old unemployed farm worker presented to his local hospital with ill health of several weeks' duration, weight loss, anorexia, and vomiting. Initial examination showed wasting and hepatomegaly and investigations showed anaemia (haemoglobin $11 \mathrm{~g} / \mathrm{dl}$ ) and an erythrocyte sedimentation rate of $120 \mathrm{~mm}$ in one hour. Further investigation was advised but the patient declined it. One month later he returned after an episode of central chest pain and severe dyspnoea. In the intervening month he had noticed increasing breathlessness. On examination there were signs of biventricular failure, a pericardial rub, and an apical diastolic murmur. Ultrasound examination showed a mass in the left atrium.

The patient was transferred to the regional cardiac unit, where examination showed an ill, thin man with peripheral oedema. His pulse was 110 beats/min and regular, his blood pressure $110 / 90 \mathrm{~mm} \mathrm{Hg}$. The central venous pressure was raised and the left ventricular impulse tapping. There was a loud pericardial rub and a loud mitral diastolic murmur. The liver was enlarged $6 \mathrm{~cm}$ below the costal margin. The electrocardiogram showed sinus rhythm with evidence of left atrial hypertrophy. The QRS complexes were of small amplitude and there were widespread nonspecific ST segment and T wave changes. There were bilateral pleural effusions on the chest radiograph. The ascending aorta appeared prominent. The two dimensional echocardiogram (Hewlett-Packard 77020A) showed an enlarged right ventricle and a small left ventricle (figs 1 and 2). There was a large echo free space surrounding the ventricles. A large mobile mass occupied the left atrium, prolapsing through the mitral valve in diastole. No pedicle or attachment to the interatrial septum was found. No tumour was seen in the right heart chambers but the right atrium

Address for reprint requests: Dr O Ormerod, Regional Cardiac Unit, Papworth Hospital, Papworth Everard, Nr Cambridge.

Accepted 7 June 1984 appeared to be indented by the "effusion" in ventricular systole. The echo free space extended anterior to the right ventricular outflow tract but no other abnormality of the great vessels was seen. A diagnosis of left atrial myxoma and pericardial effusion was made. The latter seemed remarkable, but in the presence of severe biventricular cardiac failure, pleural effusions, and hypoalbuminaemia this was attributed to his general condition. The patient was referred for resection of the tumour.

At operation, no pericardial effusion was found but the pericardial cavity was full of blood clot. There were extensive pericardial adhesions. There was a large extracardiac tumour occupying the space between the superior vena cava and the aorta. The tumour was distorting and eroding the right atrium but there was no tumour in the right heart cavities. The right atrium and pulmonary artery were enlarged. A large endothelialised tumour was found occupying the entire left atrial cavity, almost totally obstructing the mitral valve and protruding into the left ventricular cavity. The tumour extended through the roof of the left atrium and was continuous with the extracardiac tumour already noted.

It was clear that the tumour was malignant and complete resection was not possible. Frozen section confirmed the presence of a malignant sarcoma. Palliative surgical treatment was attempted by resecting as much intracardiac tumour as possible, and this was carried out with diathermy until the pulmonary veins and mitral valve were free of tumour. Postoperative echocardiographic examination showed clearing of the effusion and successful removal of the mobile left atrial tumour.

We believe that the patient presented first with symptoms due to his malignancy and hepatomegaly secondary $\delta$ to right ventricular failure from pulmonary hypertension. $\frac{3}{3}$ The presence of an effusion on the preoperative echocar-

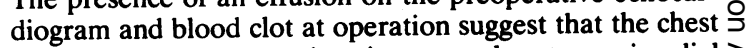
pain and sudden deterioration was due to pericardial $\frac{D}{O}$ haemorrhage. The pericardial tumour found at operation would seem to be the likely origin of the haemorrhage. $\mathrm{N}$

Subsequently the patient required surgical excision of a $\mathrm{G}$ large cutaneous secondary deposit and underwent $\mathrm{O}$ radiotherapy. His condition continued to deteriorate, how- N ever, and he died at home three months after surgery.

\section{Discussion}

Angiosarcoma of the heart is a rare tumour. There are some 70 published cases, ${ }^{4}$ but only a few where the diag- $\frac{T}{T}$ nosis was reached during life..$^{5}$ It is almost exclusively $\mathrm{a} \stackrel{+}{\vec{D}}$ tumour of the right heart; a primary left heart tumour is exceptional. ${ }^{7}$ This case is remarkable in that, although the $\mathbb{D}$ tumour affected much of the base of the heart and great 


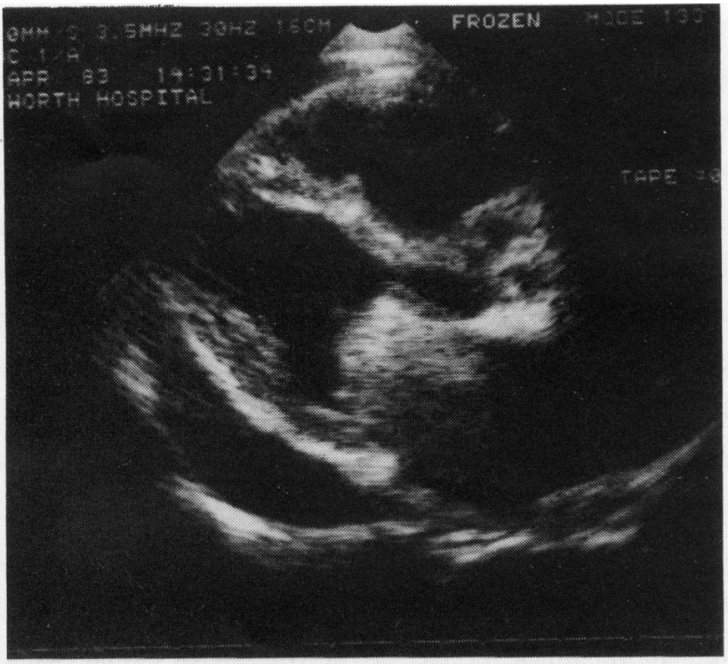

Fig 1 Preoperative two dimensional echocardiogram: the long axis view shows a large mass occupying the left atrium and the orifice of the mitral valve; the heart is surrounded by an extensive echo free space suggesting a pericardial effusion.

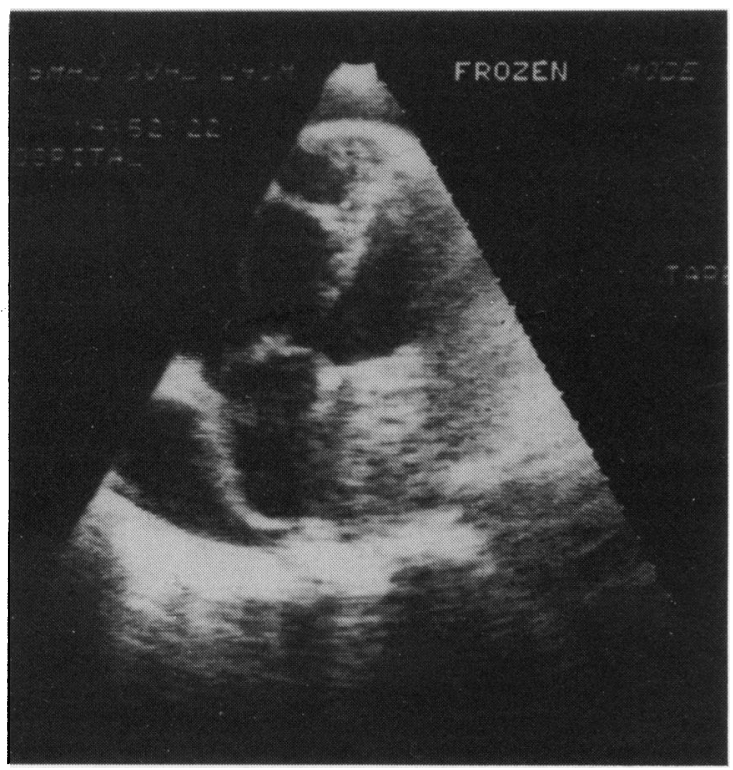

Fig 2 Preoperative two dimensional echocardiogram: systolic frame from an apical four chamber view; the left atrial mass is seen to occupy most of the left atrial cavity. vessels, the clinical picture was one of a mobile left atrial tumour mimicking a myxoma.

The uniformity of the clinical picture of angiosarcoma has been emphasised. ${ }^{3}$ As a right sided tumour it usually produces symptoms and signs of right heart obstruction, such as dyspnoea, epigastric discomfort, and a raised venous pressure, or of pericardial disease-chest pain and tamponade. Our patient did present with this picture, but his right ventricular failure was secondary to mitral valve obstruction. The two dimensional echocardiogram showed an apparently mobile mass in the left atrium, obstructing the mitral valve orifice. The large pericardial effusion and pericardial rub noted on clinical examination were attributed to severe heart failure. The echocardiogram was so characteristic of a left atrial myxoma that resection of the tumour was recommended without hesitation. In retrospect there are features of the echocardiogram that are unusual as an effusion is not usually seen with myxomas. Haemopericardium is a feature of pericardial angiosarcoma noted in previous reports ${ }^{38}$; this is undoubtedly the explanation of the pericardial effusion seen on the echocardiogram in this case. It has, however, previously been reported that pericardial invasion with tumour may give rise to an apparent pericardial effusion on the echocardiogram. ${ }^{8}$ This phenomenon may explain why the extent of the tumour found at operation was underestimated by the echocardiogram.

In conclusion, the presentation in this case was unique for this rare, normally right sided tumour, closely mimicking a left atrial myxoma. Although surgical exploration of the heart is warranted to attempt to relieve the severe mitral valve obstruction, the presence of pericardial disease and apparent pericardial effusion should suggest that a malignant process may be responsible and that invasion by the tumour may be more extensive than the echocardiogram suggests.

\section{References}

' Heath D. Pathology of cardiac tumours. Am J Cardiol 1968;21:315-27.

${ }^{2}$ Freeland JP, Sy BG, Ahluwalis MS, Dunea G. Hemangiosarcoma of the heart. Chest 1971;60:222-4.

${ }^{3}$ Hollingsworth JH, Sturgill BC. Treatment of primary angiosarcoma of the heart. Am Heart J 1969;78:254-8.

${ }^{4}$ Lin TK, Stech JM, Eckert WG, Lin JJ, Farha SJ, Hagan CT. Pericardial angiosarcoma simulating pericardial effusion by echocardiography. Chest 1978;73:881-3.

${ }^{5}$ Shackell M, Mitko A, Williams PL, Sutton GC. Angiosarcoma of the heart. Br Heart J 1979;41:498-503.

- Gross P, Englehart CE. Primary hemangioendothelioma of the heart: report of a case. Am J Cancer 1937;30:103.

' Hager W, Kremer K, Muller W. Angiosarkom des Herzens. Dische Med Wochenschr 1970;95:680.

${ }^{8}$ Panella JS, Paige ML, Victor TA, Semerdjian RA, Hueter DC. Angiosarcoma of the heart. Diagnosis by echocardiography. Chest 1979;76:221-3. 